\title{
Pembrolizumab-Induced Meningoencephalitis: A Brain Autopsy Case
}

\author{
Seigo Minami ${ }^{\text {a, d }}$, Hideyasu Okada ${ }^{\text {a }}$, Shoichi Ihara ${ }^{\text {a }}$, Hiromi Tsuji ${ }^{\mathrm{b}}$, \\ Misaki Yamadera ${ }^{\mathrm{c}}$, Hironao Yasuoka ${ }^{\mathrm{b}}$
}

\begin{abstract}
Encephalitis is very rare, but often fatal immune-related adverse event (irAE) of immune checkpoint inhibitors (ICIs). A 65-year-old Japanese woman was admitted to our hospital because of general fatigue, chillness and high-grade fever for 4 days, 8 months after the initiation of the first-line pembrolizumab monotherapy for metastatic pulmonary adenocarcinoma. On the hospital day 3 , she suddenly presented delirium and uncontrollable impaired consciousness. Although the magnetic resonance imaging (MRI) did not suggest a diagnosis of encephalitis and meningitis, the spinal fluid showed abnormally elevated levels of protein $(317.6 \mathrm{mg} / \mathrm{L})$ and cell count $(197$ cells $/ \mu \mathrm{L})$ with increased mononuclear cells (93\%). An empirical and intravenous administration of acyclovir in doses of $10 \mathrm{mg} / \mathrm{kg}$ body weight every $8 \mathrm{~h}$ and steroid pulse therapy in dose of $1 \mathrm{~g} /$ body/day from the hospital day 5 until her death failed to improve her conditions. She died on the hospital day 8 . The postmortem autopsy showed viable cancer cells in the metastatic tumor in the left occipital lobe and in the spinal fluids. However, many inflammatory cells infiltration in the meninges and perivascular cuffing were prominent especially in the brain stem and medial part of the temporal lobe. Infiltrating lymphocytes in the meninges and parenchyma of the brain stem were mainly composed of cluster of differentiation (CD)8-positive lymphocytes. For irAE encephalitis, early recognition of early signs and symptoms and subsequent early therapeutic intervention are necessary. It is important for oncologists to keep in mind of the possible adverse effects of immunotherapies on the nervous system.
\end{abstract}

Keywords: Pembrolizumab; Immune checkpoint inhibitor; Immunerelated adverse event; Meningoencephalitis; Brain autopsy; Highgrade fever; Non-small cell lung cancer; Lymphocyte infiltration

Manuscript submitted July 18, 2021, accepted August 3, 2021

Published online August 25, 2021

aDepartment of Respiratory Medicine, Osaka Police Hospital, 10-31 Kitayama-cho, Tennoji-ku, Osaka City, Osaka 543-0035, Japan

bDepartment of Pathology, Osaka Police Hospital, 10-31 Kitayama-cho, Tennoji-ku, Osaka City, Osaka 543-0035, Japan

'Department of Pathology, National Hospital Organization Osaka Toneyama Medical Center, Toyonaka, Osaka, Japan

${ }^{\mathrm{d}}$ Corresponding Author: Seigo Minami, Department of Respiratory Medicine, Osaka Police Hospital, 10-31 Kitayama-cho, Tennoji-ku, Osaka City, Osaka 543-0035, Japan. Email: seigominami@oph.gr.jp

doi: https://doi.org/10.14740/jmc3748

\section{Introduction}

Immune checkpoint inhibitors (ICIs) are widely used in the treatment of various cancers, including various clinical settings of advanced non-small cell lung cancer (NSCLC). Pembrolizumab is a humanized monoclonal antibody for the programmed cell death protein 1 (PD-1) receptor of lymphocytes, and immunologically enhances antitumor activity by activating $\mathrm{T}$ cells. There are an abundant accumulation of case reports on immunerelated adverse events (irAEs) of cancer immunotherapies on the nervous system. According to a retrospective review of 3,763 patients with advanced melanoma treated with nivolumab alone or nivolumab plus ipilimumab, there were a total of six cases of encephalitis (overall incidence of $0.16 \%$ ), including two lifethreatening cases and one fatal case [1]. An irAE of encephalitis is rare, but usually severe and potentially fatal. However, the diagnosis of irAE encephalitis may be challenging. Most case reports of ICIs-induced encephalitis were clinically diagnosed without histological analyses of brain sampling. The etiology of this irAE remains unknown, though there are some hypotheses for the mechanisms, for example, autoimmune paraneoplastic disorders triggered by ICIs or manifestation of latent autoimmunity unmasked by ICIs [2]. Herein, we report a brain autopsy case of a patient who developed meningoencephalitis during pembrolizumab monotherapy for pulmonary adenocarcinoma.

\section{Case Report}

\section{Investigations}

A 65-year-old Japanese woman visited a gastroenterologist because of nausea, vomiting, anorexia and gait disorder. She had diabetes mellitus, hypertension, hyperlipidemia and alcoholism. A chest computed tomography (CT, Fig. 1a) and brain magnetic resonance imaging (MRI, Fig. 1b) detected a mass of $5.5 \mathrm{~cm}$ in diameter in the left lower lobe with multiple pulmonary nodules and multiple brain tumors in the parietal lobe and left cerebellum. She was referred to our department. Bronchoscopic tumor biopsy diagnosed her tumor as adenocarcinoma. We could not find any driver mutations in epidermal growth factor receptor (EGFR), anaplastic lymphoma kinase (ALK) and ROS-1, but immunohistologically detected high expression of tumor proportion score (TPS) $90-100 \%$ of PD- 

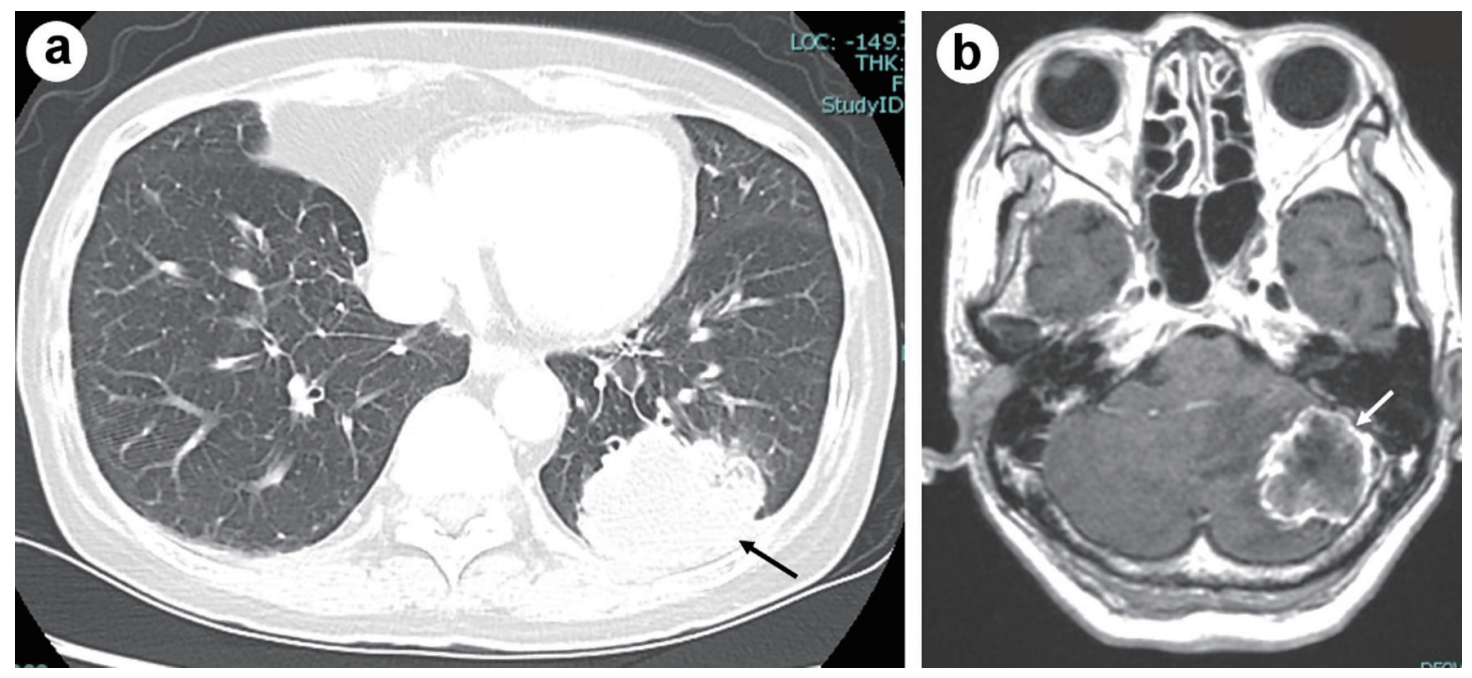

Figure 1. Chest computed tomography imaging of the primary tumor in the left lower lobe (shown by black arrow) before the start of pembrolizumab (a), and brain magnetic resonance imaging (MRI) of metastasis in the cerebellum (shown by white arrow) in T1-weighted image with gadolinium enhancement at the first visit to our hospital in March 2018 (b).

L1 (clone 22C3, Dako). She received a surgery to remove the biggest tumor of $3.5 \mathrm{~cm}$ in diameter and its surrounding edema in the left cerebellum at the risk of brain hernia. The pathological analysis of the cerebellar tumor revealed a metastatic adenocarcinoma originated from lung cancer. As a result of systemic screening, her staging was c-stage IVB (cT4N3M1c) with multiple metastases in bilateral lungs, adrenal glands, brain and subcutaneous region of the anterior chest wall. She started pembrolizumab monotherapy in May 2018.

\section{Diagnosis}

After 13 cycles of pembrolizumab, 8 months after the initiation of pembrolizumab, she presented general fatigue, chillness and then high-grade fever of $40.0^{\circ} \mathrm{C}$. She visited our emergency room and was admitted to our department; 4 days after the initiation of the fever. A chest and abdominal CT screening found neither an origin of the persistent fever nor regrowth of the primary and metastatic tumors (Fig. 2a). Except for hyponatremia $(128 \mathrm{mEq} / \mathrm{L})$, blood tests and cultures did not detect viral, bacterial or fungal infection. Leukocyte counts $(9,200 /$ $\mu \mathrm{L})$ and C-reactive protein $(0.06 \mathrm{mg} / \mathrm{dL})$ were within normal range. Until the hospital day 3, she was exhausted with the high fever, but kept clear consciousness. Administration of acetaminophen failed to suppress her fever. We did not find nuchal stiffness at that time. At the night of the hospital day 3 , she suddenly presented delirium together with a high fever of
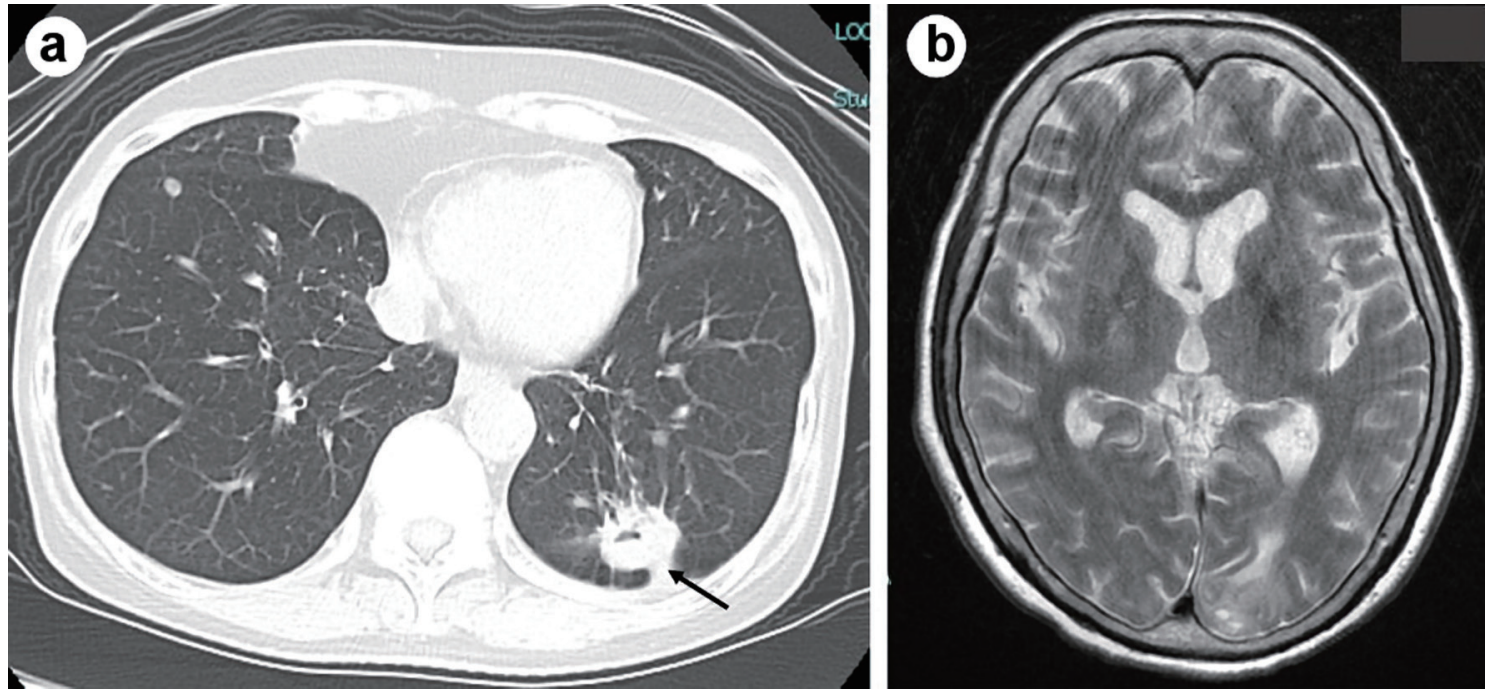

Figure 2. Chest computed tomography imaging did not show regrowth of the primary tumor in the left lower lobe (shown by black arrow) on admission (a). Brain magnetic resonance imaging (MRI) in plain T2-weighted image on the hospital day 4 did not suggest a diagnosis of encephalitis and meningitis (b). 


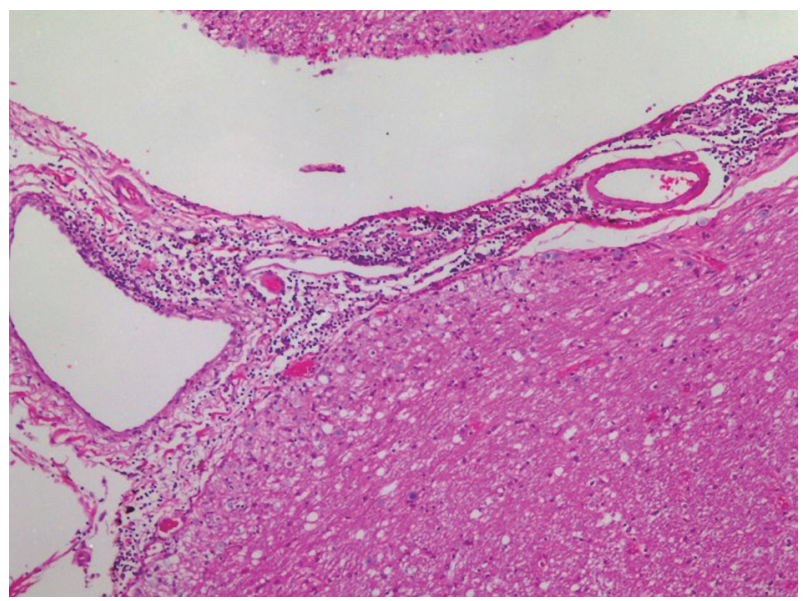

Figure 3. Medulla oblongata obtained through autopsy: infiltration of lymphocytes, plasma cells and macrophages in the meninges (Hematoxylin and Eosin stain).

$40.0^{\circ} \mathrm{C}$. Thereafter, her high-grade fever and impaired level of consciousness became uncontrollable. On the hospital day 4 , her nuchal stiffness and convulsions appeared. Despite of artifacts arising from her body movements, the MRI did not suggest a diagnosis of encephalitis and meningitis (Fig. 2b). The colorless and transparent spinal fluid obtained by lumbar puncture on the hospital day 5 showed elevated levels of protein $(317.6 \mathrm{mg} / \mathrm{L})$ and cell count $(197$ cells $/ \mu \mathrm{L})$ with a predominance of mononuclear cells (93\%), but within normal range of glucose $(55.0 \mathrm{mg} / \mathrm{mL})$ and adenosine deaminase (ADA) $(7.7$ $\mathrm{IU} / \mathrm{L})$. The PCR method and culture did not detect DNA of herpes simplex virus and bacterial infection in the fluid. Cancer cells were detected in the spinal fluids. On the basis of MRI and spinal fluid findings, we suspected carcinomatous, viral, tuberculous or fungal meningitis as a differential diagnosis.

\section{Treatment}

An empirical and intravenous administration of acyclovir in doses of $10 \mathrm{mg} / \mathrm{kg}$ body weight every $8 \mathrm{~h}$ and steroid pulse therapy in dose of $1 \mathrm{~g} /$ body/day from the hospital day 5 until her death failed to improve her conditions.

\section{Follow-up and outcomes}

She died on the hospital day 8 . We performed an autopsy approximately $8 \mathrm{~h}$ after her death. The brain weight was 1,539 g. The gross anatomic examination showed no abnormalities, except for the previous surgical scar in the cerebellum. Viable cancer cells existed in the surrounding area of the central necrotic lesion in the metastatic tumor in the left occipital lobe. Meningeal carcinomatosis was not indicated histopathologically. Perivascular cuffing and infiltration of lymphocytes, plasma cells, and macrophages in the meninges were prominent especially in the brain stem and medial temporal lobe (Fig. 3). Perivascular lymphocytes infiltration was mainly composed of cluster of differentiation (CD)3 (clone 2GV6, Roche)positive or CD20 (clone L26, Roche)-positive lymphocytes. On the other hand, in the parenchyma of the brain stem, there were CD3-positive lymphocytes. Among these CD3-positive lymphocytes, there were many CD8 (clone C8/114B, Dako)positive lymphocytes and small numbers of CD4 (clone 4B12, Nichirei)-positive lymphocytes (Fig. 4). Except for very small number of cancer cells in the lymphatic ducts in lungs, the primary tumor and metastatic nodules in her lung did not contain viable cancer cells, and were replaced with necrosis or fibrosis. Infiltration of many inflammatory cells and aggregation of bacteria suggested bronchopneumonia in the bilateral lower lobes. Metastatic cancer cells appeared in the bilateral adrenal glands, the tunica adventitia of esophagus, and the surrounding lymph nodes of esophagus and pancreas.

\section{Discussion}

To our knowledge, there were previously three brain autopsy cases of encephalitis due to ICIs (Table 1 [3-5]). Among these four fatal cases [3-5], there were some clinical and pathological differences in the initial symptom, in the timing of occurrence of encephalitis from the initiation of ICI (17 days to 8 months), in the interval from symptom presentation to death (10 to 40 days), in the association of paraneoplastic syndrome (PNS) [3], and in the formation of necrotic lesion in the autopsy brains [4]. On the other hand, there was a common finding in the damages in brain stem, i.e., critically life-threatening area and in the type of infiltrating lymphocytes, i.e., mainly CD8-positive T cells. Their deaths were considered to be due to autoimmune-mediated brainstem encephalitis, which had damaged cardiovascular and respiratory centers. Compared with the other three cases, our case showed delayed onset from the initiation of pembrolizumab (8 months), but more rapid course of deterioration (only 10 days from symptom presentation to death). If our case had been slowly progressive and more aggressively treated, regression of lymphocyte infiltration and histological change into necrotic tissue might have been observed in the brain, as shown in the case reported by Leitinger et al [4].

Encephalitis as irAE clinically presented various clinical courses. Reviewing the previous cases of irAE encephalitis in NSCLC (Table 2 [3, 4, 6-18]), there were differences in symptoms, timing of occurrence, treatments, response to treatments, PNS antibodies and cerebrospinal fluid (CSF) findings. It may be very difficult for oncologists to suspect encephalitis as irAE. We hesitated to use steroid when the initial symptom was only high-grade fever. In fact, our autopsy suggested that her fever might have been caused mainly by meningoencephalitis, but partially by bronchopneumonia. When we suspect irAE encephalitis, early introduction of steroid pulse or highdose steroid therapy is recommended.

Our case included two limitations. First, we did not examine the serum or CSF antibody tests for PNS, and could not discuss the association of PNS with irAE meningoencephalitis. Second, we could not deny the association of brain metastasis and carcinomatous meningitis with her rapid deteriorated 

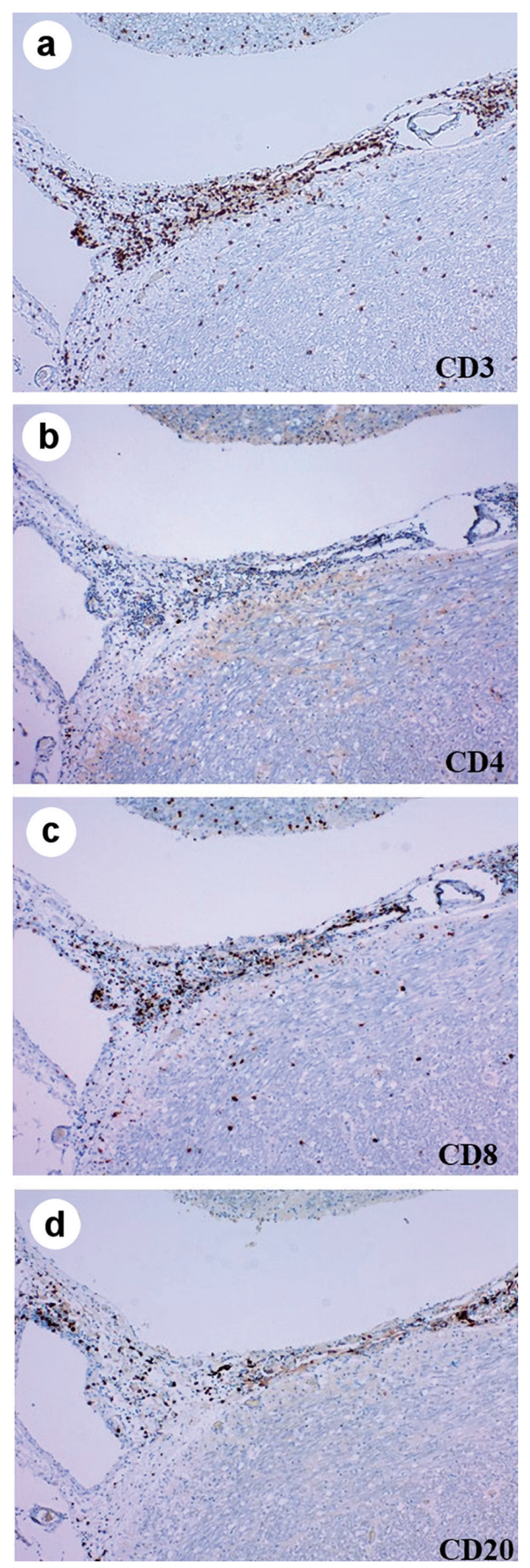

Figure 4. Immunohistochemistry of medulla oblongata stained by antiCD3 (a), CD4 (b), CD8 (c) and CD20 (d) antibodies. CD: cluster of differentiation.

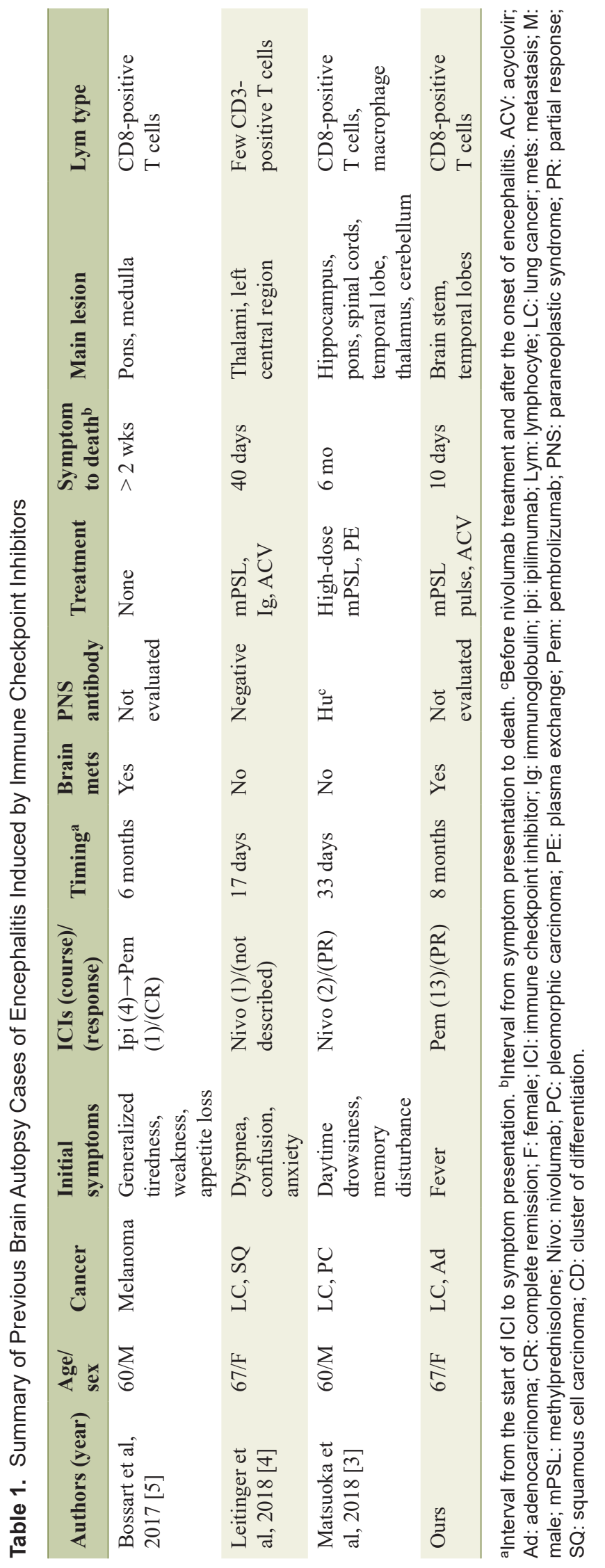




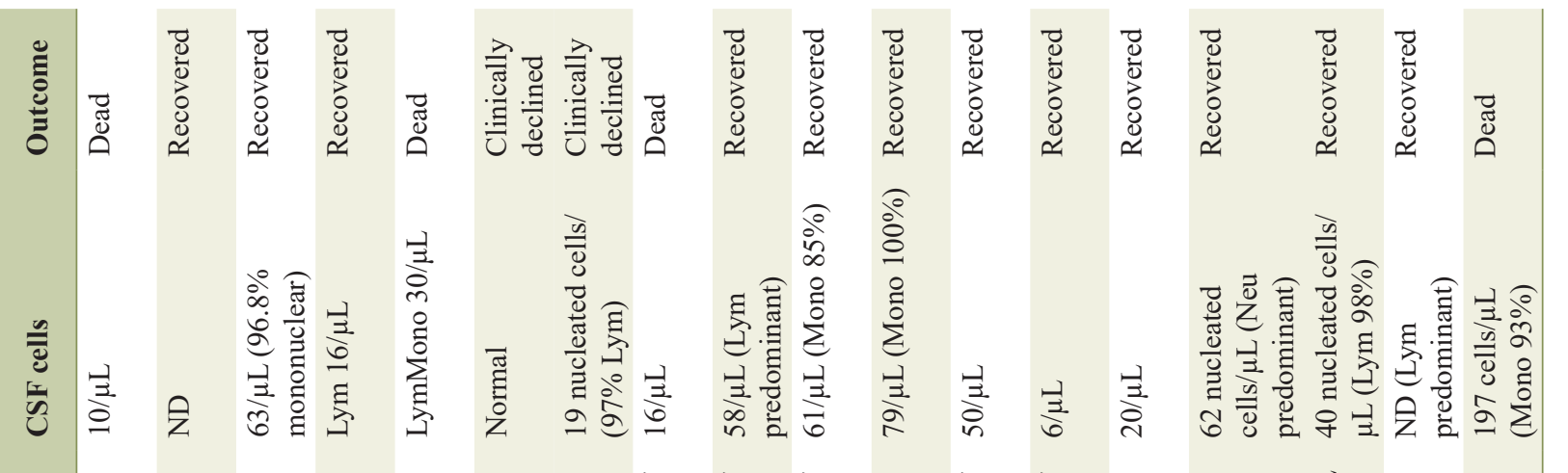

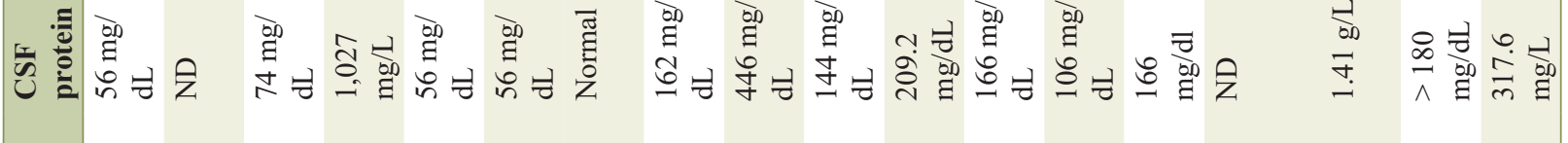

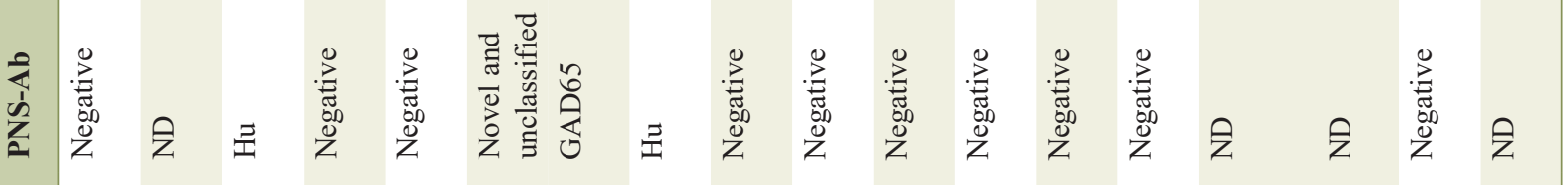

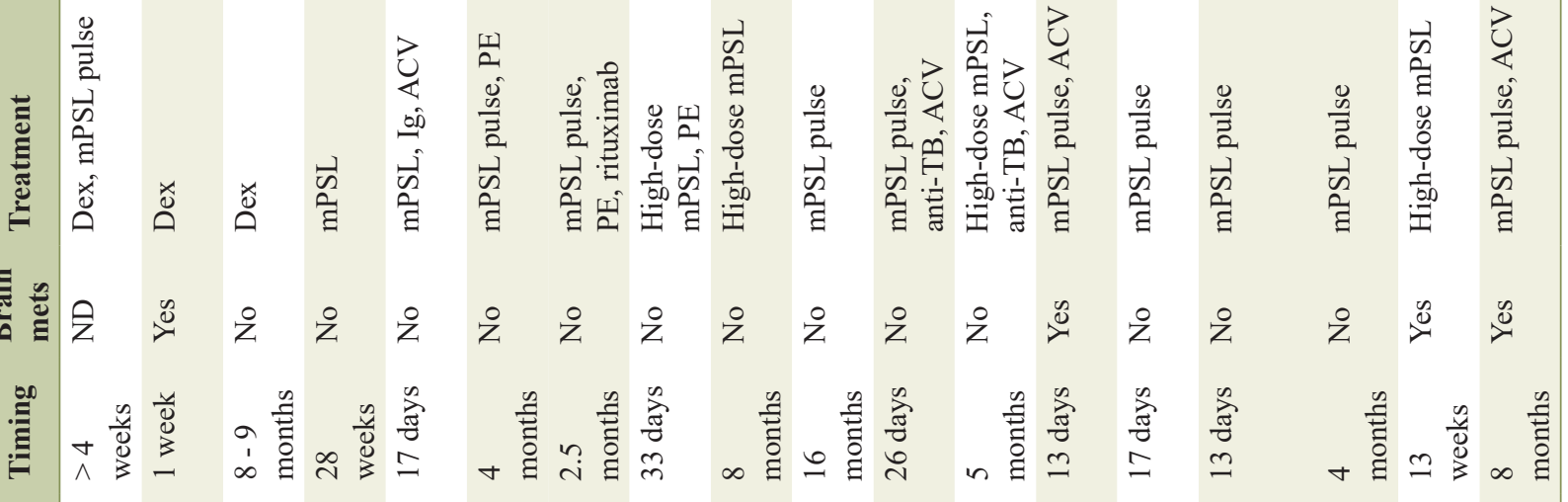

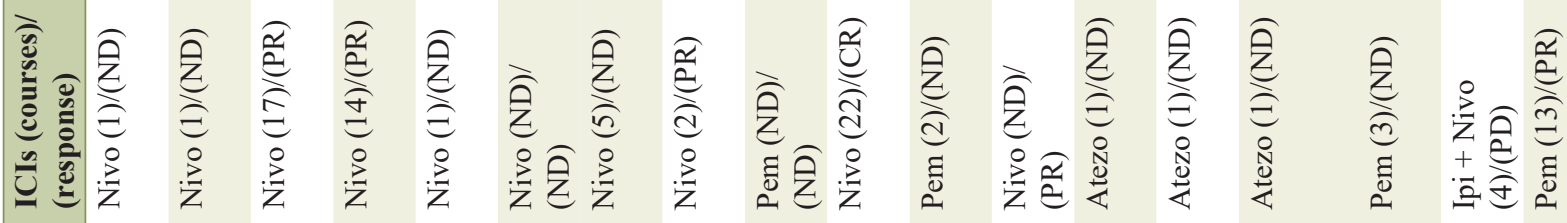

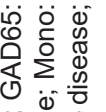

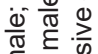

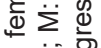
نे 总高

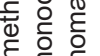

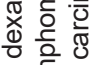

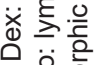

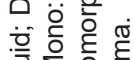

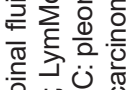

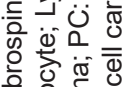
要 응 은 은

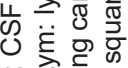

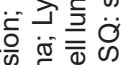

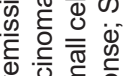

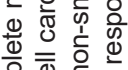
흘

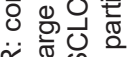

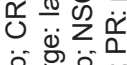

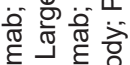
총 3

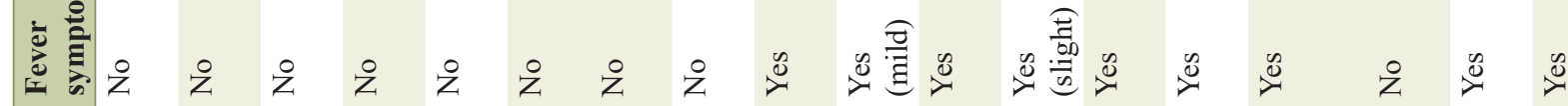

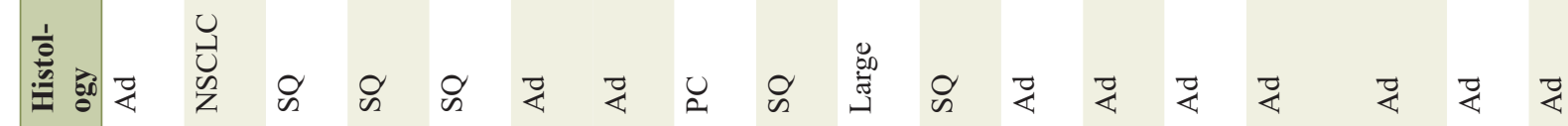

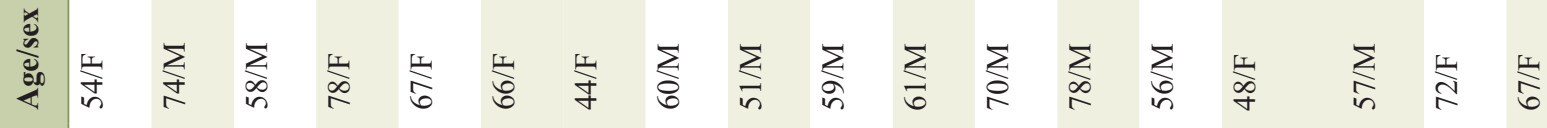
के 
conditions. Considering the brain autopsy, we strongly suspect irAE meningoencephalitis as a major etiology.

\section{Conclusions}

Encephalitis is often fatal irAE. Early recognition of early signs and symptoms and subsequent early therapeutic intervention are necessary. Therefore, it is important for oncologists to keep in mind of the possible adverse effects of immunotherapies on the nervous system.

\section{Acknowledgments}

None to declare.

\section{Financial Disclosure}

None to declare.

\section{Conflict of Interest}

None to declare.

\section{Informed Consent}

Not applicable because the manuscript has been sufficiently de-identified to protect the patient.

\section{Author Contributions}

Minami S, Ihara $\mathrm{S}$ and Okada $\mathrm{H}$ were mainly involved in diagnosis and treatment of this patient. Tsuji H, Yamadera $M$ and Yasuoka $\mathrm{H}$ were involved in pathological diagnosis and autopsy. Minami S drafted the report. All authors read and critically reviewed the manuscript, and then approved the final submitted version.

\section{Data Availability}

The authors declare that data supporting the findings of this study are available within the article.

\section{References}

1. Larkin J, Chmielowski B, Lao CD, Hodi FS, Sharfman W, Weber J, Suijkerbuijk KPM, et al. Neurologic serious adverse events associated with nivolumab plus ipilimum$\mathrm{ab}$ or nivolumab alone in advanced melanoma, including a case series of encephalitis. Oncologist. 2017;22(6):709718.
2. Valencia-Sanchez C, Zekeridou A. Paraneoplastic neurological syndromes and beyond emerging with the introduction of immune checkpoint inhibitor cancer immunotherapy. Front Neurol. 2021;12:642800.

3. Matsuoka H, Kimura H, Koba H, Tambo Y, Ohkura N, Hara J, Sone T, et al. Nivolumab-induced limbic encephalitis with anti-hu antibody in a patient with advanced pleomorphic carcinoma of the lung. Clin Lung Cancer. 2018;19(5):e597-e599.

4. Leitinger M, Varosanec MV, Pikija S, Wass RE, Bandke D, Weis S, Studnicka M, et al. Fatal Necrotizing encephalopathy after treatment with nivolumab for squamous non-small cell lung cancer: case report and review of the literature. Front Immunol. 2018;9:108.

5. Bossart S, Thurneysen S, Rushing E, Frontzek K, Leske $\mathrm{H}$, Mihic-Probst D, Nagel HW, et al. Case report: encephalitis, with brainstem involvement, following checkpoint inhibitor therapy in metastatic melanoma. Oncologist. 2017;22(6):749-753.

6. Kawamura R, Nagata E, Mukai M, Ohnuki Y, Matsuzaki T, Ohiwa K, Nakagawa T, et al. Acute cerebellar ataxia induced by nivolumab. Intern Med. 2017;56(24):3357-3359.

7. Richard K, Weslow J, Porcella SL, Nanjappa S. A case report of steroid responsive nivolumab-induced encephalitis. Cancer Control. 2017;24(5):1073274817729069.

8. Raskin J, Masrori P, Cant A, Snoeckx A, Hiddinga B, Kohl S, Janssens A, et al. Recurrent dysphasia due to nivolumab-induced encephalopathy with presence of $\mathrm{Hu}$ autoantibody. Lung Cancer. 2017;109:74-77.

9. Schneider S, Potthast S, Komminoth P, Schwegler G, Bohm S. PD-1 checkpoint inhibitor associated autoimmune encephalitis. Case Rep Oncol. 2017;10(2):473-478.

10. Shah S, Dunn-Pirio A, Luedke M, Morgenlander J, Skeen M, Eckstein C. Nivolumab-induced autoimmune encephalitis in two patients with lung adenocarcinoma. Case Rep Neurol Med. 2018;2018:2548528.

11. Niki M, Nakaya A, Kurata T, Nakahama K, Yoshioka H, Kaneda T, Kibata K, et al. Pembrolizumab-induced autoimmune encephalitis in a patient with advanced nonsmall cell lung cancer: A case report. Mol Clin Oncol. 2019;10(2):267-269.

12. Miller PDE, Snowden JA, De Latour RP, Iacobelli S, Eikema DJ, Knol C, Marsh JCW, et al. Autoimmune cytopenias (AIC) following allogeneic haematopoietic stem cell transplant for acquired aplastic anaemia: a joint study of the Autoimmune Diseases and Severe Aplastic Anaemia Working Parties (ADWP/SAAWP) of the European Society for Blood and Marrow Transplantation (EBMT). Bone Marrow Transplant. 2020;55(2):441-451.

13. Yonenobu Y, Ishijima M, Toyooka K, Fujimura H. [A case of meningoencephalitis associated with pembrolizumab treated for squamous cell lung cancer]. Rinsho Shinkeigaku. 2019;59(2):105-108.

14. Fujiwara S, Mimura N, Yoshimura H, Fujimoto D, Ito $\mathrm{M}$, Mori R, Ito J, et al. Elevated adenosine deaminase levels in the cerebrospinal fluid in immune checkpoint inhibitor-induced autoimmune encephalitis. Intern Med. 2019;58(19):2871-2874.

15. Arakawa M, Yamazaki M, Toda Y, Saito R, Ozawa A, Ko- 
saihira S, Kimura K. Atezolizumab-induced encephalitis in metastatic lung cancer: a case report and literature review. eNeurologicalSci. 2019;14:49-50.

16. Yamaguchi Y, Nagasawa H, Katagiri Y, Wada M. Atezolizumab-associated encephalitis in metastatic lung adenocarcinoma: a case report. J Med Case Rep. 2020;14(1):88.

17. Robert L, Langner-Lemercier S, Angibaud A, Sale A,
Thepault F, Corre R, Lena H, et al. Immune-related encephalitis in two patients treated with immune checkpoint inhibitor. Clin Lung Cancer. 2020;21(5):e474-e477.

18. Singh V, Zhao CW, Gupta V, Chu Y. Combination Checkpoint Inhibitor-induced Antibody Negative Autoimmune Encephalitis in Non-small Cell Lung Cancer. Neurol India. 2021;69(1):222-227. 